\title{
ON SOME THEOREMS OF BÔCHER CONCERNING ISOLATED SINGULAR POINTS OF HARMONIC FUNCTIONS*
}

BY O. D. KELLOGG

In a note entitled Deux théorèmes élémentaires sur les singularités des fonctions harmoniques, $\uparrow$ Picard gave proofs of the following theorems.

I. If $u(x, y)$ is single-valued and harmonic in the neighborhood of a point $A$, except at $A$, and is bounded, then $u(x, y)$ becomes harmonic at $A$ also when its definition at this point is properly adjusted.

II. If $u(x, y)$ is single-valued and harmonic in the neighborhood of $A$, except at $A$, and becomes positively infinite at $A$, then $u(x, y)-h \log (1 / r)$ is harmonic at $A$, where $h$ is a properly chosen constant, and $r$ is the distance of $(x, y)$ from $A$.

As credit for these results was not assigned in the note cited, the author must have been unaware of a paper by Bôcher, $\ddagger$ in which were proved the above theorems, and several others, not only for the plane, but for $n$ dimensions, and for other differential equations of the elliptic type as well as Laplace's.

In addition to calling attention to the above matter of authorship, the object of this paper is to give an elementary proof of a theorem from which follow at once the results given by Bôcher, as far as solutions of Laplace's equation

* Presented to the Society, September 9, 1926. See the concluding footnote.

† Comptes Rendus, vol. 176 (1923), pp. 933-35.

$\ddagger$ Singular points of functions which satisfy partial differential equations of the elliptic type, this BulletiN, vol. 9 (1903), pp. 455-465. 
are concerned. We shall reason in three dimensions; the same argument is available for the corresponding theorems in any space of two or more dimensions.

We first derive a result of which a special case has been given by Lebesgue.* Here, and in the following, we shall use $N$ to denote a neighborhood of the point $O$ from which $O$ has been removed. The harmonic functions are all assumed to be one-valued.

THEOREM I. Let $U(P)$ be harmonic in $N$, and such that there exists a positive integer, $m$, for which

$$
\lim _{r \rightarrow \infty} r^{m} U(P)=0,
$$

uniformly. Then in $N$,

(1) $U(P)=V(P)+\frac{S_{0}(\phi, \theta)}{r}+\frac{S_{1}(\phi, \theta)}{r^{2}}+\cdots+\frac{S_{m-2}(\phi, \theta)}{r^{m-1}}$,

where $r^{i} S_{i}(\phi, \theta)$ is the usual spherical harmonic of order $i$, and $V(P)$ is harmonic throughout a neighborhood of $O$.

The existence of the infinite development for $U(P)$ corresponding to (1) is well known; also the uniform convergence of this development on all spheres about $O$ of sufficiently small positive radius :

$$
U(P)=\theta \delta(r) / r^{m}=V(P)+\frac{S_{0}(\phi, \theta)}{r}+\frac{S_{1}(\phi, \theta)}{r^{2}}+\cdots .
$$

Our hypothesis is contained in this formula if it is understood that $|\Theta| \leqq 1$, and that $\delta(r) \rightarrow 0$ with $r$.

The coordinates of $P$ are $(r, \phi, \theta)$. Let both members of the equation (2) be multiplied by $P_{n}(\cos \gamma) \sin \theta$, and integrated from 0 to $\pi$ with respect to $\theta$, and from 0 to

* Sur les singularités des fonctions harmoniques, Comptes Rendus, vol. 176 (1923), pp. 1270-71. 
$2 \pi$ with respect to $\phi$, where $\cos \gamma=\cos \theta \cos \theta^{\prime}+\sin \theta \sin \theta^{\prime}$ $\cos \left(\phi-\phi^{\prime}\right)$. The result is

(3) $\frac{\delta(r)}{r^{m}} \iint \theta(r, \phi, \theta) P_{n}(\cos \gamma) \sin \theta d \theta d \phi$

$=\iint V(r, \phi, \theta) P_{n}(\cos \gamma) \sin \theta d \theta d \phi+\frac{4 \pi}{2 n+1} \cdot \frac{S_{n}\left(\phi^{\prime}, \theta^{\prime}\right)}{r^{n+1}}$.

If, now, we multiply the terms of this equation by $r^{n+1}$, and allow $r$ to approach 0 , we see that if $n-m+1 \geqq 0$, $n \geqq 0, S_{n}\left(\phi^{\prime}, \theta^{\prime}\right) \equiv 0$. Thus the equation (1) is established.

Theorem II. Let $U(P)$ be harmonic in $N$, and be either bounded above, or bounded below. Then in $N$

$$
U(P)=c / r+V(P),
$$

where $V(P)$ is harmonic throughout a neighborhood of $0 . *$

The proof is based on inequalities of Harnack $\nmid$ for positive harmonic functions, which, in three-dimensional space, take the form

$$
\frac{(R-r) R}{(R+r)^{2}} U(C) \leqq U(P) \leqq \frac{(R+r) R}{(R-r)^{2}} U(C)
$$

where $U(P)$ is harmonic and not negative throughout the sphere of radius $R$ and with $C$ as center, and where $r=C P$.

We may reduce our problem to one in which $U(P) \geqq 0$ in $N$ by a change of sign and the addition of a constant, if necessary. Now let $a$ be a positive number such that a sphere of radius $2 a$ about $O$ contains, with the exception of $O$, only points of $N$, and let $M$ be the maximum of $U(P)$ on a sphere of radius $a$ about $O$. Let $\theta$ be a constant between 0 and 1 . We apply the second inequality (4) to $U(P), C$

* This theorem is a direct consequence of the theorems of Bocher, V and II, loc. cit., pp. 461 and 456.

$\dagger$ Theorie des logarithmischen Potentials, Leipzig, 1887, p. 62. 
being on the sphere of radius $a$ about $O, R$ being $\theta a$, and $r$ being $\theta R$, or $\theta^{2} a$. The result is

$$
U(P) \leqq \frac{1+\theta}{(1-\theta)^{2}} M, \text { for } a\left(1-\theta^{2}\right) \leqq O P \leqq a .
$$

With this bound for $U(P)$ on the sphere of radius $a\left(1-\theta^{2}\right)$ about $O$, we again apply the inequality of (4), taking $C$ on this sphere, and replacing $a$ by $a\left(1-\theta^{2}\right)$. After a repetition of this process, we obtain the general result

$$
U(P) \leqq\left[\frac{1+\theta}{(1-\theta)^{2}}\right]^{n} M, \text { for } a\left(1-\theta^{2}\right)^{n} \leqq O P \leqq a\left(1-\theta^{2}\right)^{n-1} .
$$

Let us now replace $n$ by a continuous variable, $s$, and write $r=a\left(1-\theta^{2}\right)^{s}$. Our result then yields the inequality

$$
U(P) \leqq\left[\frac{1+\theta}{(1-\theta)^{2}}\right]^{o+1} M, \text { for } n-1 \leqq s \leqq n,
$$

since the bound for $U(P)$ is an increasing function of $s$. But as this bound is independent of $n$, (5) holds for all $s \geqq 1$. If, now, we replace $s$ by its value in terms of $r$, we arrive at the result

where

$$
U(P) \leqq \frac{1+\theta}{(1-\theta)^{2}} \cdot \frac{M}{a} \cdot r^{p},
$$

$$
p=\frac{\log (1+\theta)-2 \log (1-\theta)}{\log (1+\theta)+\log (1-\theta)} .
$$

As $\theta \rightarrow 1, p$ approaches -2 from below. It follows that $U(P)$ is subject to an inequality of the type $U(P) \leqq M r^{-2-\eta}$, where $\eta$ may be any fixed positive number.

Such a function, as we have seen in Theorem I, is of the form

$$
U(P)=V(P)+c / r+S_{1}(\phi, \theta) / r^{2} .
$$

But if $S_{1}(\phi, \theta)$ were not identically 0 , it would have both positive and negative values, and hence for small enough $r, U(P)$ would have to assume arbitrarily great positive 
and negative values. But this contradicts our hypothesis. Therefore $S_{1}(\phi, \theta) \equiv 0$, and $U(P)$ has the stated form.

We close with some consequences of the above two theorems. In the first place, the theorem on isolated singularities of bounded harmonic functions (the first one quoted at the outset) is included in Theorem I.*

The second theorem quoted is a consequence of Theorem II,* for a function, harmonic in $N$, which becomes positively infinite at $O$ must be positive in some neighborhood of $O$ (with $O$ omitted).

Finally, we may also infer the theorem of Bôcher†: If a function $U(P)$ of two or more variables, harmonic in $N$, becomes infinite for some but not for all modes of approach of $P$ to 0 , then it can be made to become both positively and negatively infinite (and therefore also to take on every real value an infinite number of times) by suitable choice of the mode of approach of $P$ to 0 . For if $U(P)$ did not become both positively and negatively infinite in the neighborhood of 0 , it would, by Theorem II, become positively infinite for every mode of approach, since the fact that it becomes infinite for some mode of approach assures us that $c$ is not 0 in that theorem. $\ddagger$

HARVARD UNIVERSITY

* It being understood that the wording is suitably altered to meet the two-dimensional case.

$\dagger$ Loc. cit., footnote 3, p. 664 .

$¥$ At the time of writing this note, the author was unaware of a paper on the same subject read before the Society October 31, 1925, by G. E. Raynor, and published in the last number of the Bulletin. An earlier knowledge of Raynor's paper would have resulted in modifications of the above, but under the circumstances it seemed best to publish it unaltered and this has been done.

Although the papers are written, one for two and the other for three dimensions, the methods used are all available for any space $n \geqq 2$. Theorem II of the present note is implied by Raynor's IV (Böcher's V, loc. cit.), while Theorem II, above, is independent, and involves an additional hypothesis. 